\title{
Evaluation of an integral injection molded housing for high power density synchronous machines with concentrated single-tooth winding
}

\author{
Andreas Langheck \\ Karlsruhe Institute of Technology (KIT) \\ Institute of Electrical Engineering \\ Karlsruhe, Germany \\ andreas.langheck@kit.edu
}

\author{
Robert Maertens \\ Fraunhofer Institute for Chemical \\ Technology - ICT \\ Polymer Engineering \\ Pfinztal, Germany \\ robert.maertens@ict.fraunhofer.de
}

\author{
Steffen Reuter \\ Fraunhofer Institute for Chemical \\ Technology - ICT \\ New Drive Systems \\ Karlsruhe, Germany \\ steffen.reuter@ict.fraunhofer.de
}

Florian Wittemann

Karlsruhe Institute of Technology (KIT)

Institute of Vehicle System Technology

Karlsruhe, Germany

florian.wittemann@kit.edu

Martin Doppelbauer

Karlsruhe Institute of Technology (KIT)

Institute of Electrical Engineering

Karlsruhe, Germany

martin.doppelbauer@kit.edu

\author{
Oleg Saburow \\ Karlsruhe Institute of Technology (KIT) \\ Institute of Vehicle System Technology \\ Karlsruhe, Germany \\ oleg.saburow@kit.edu
}

Lars Fredrik Berg

Fraunhofer Institute for Chemical

Technology - ICT

New Drive Systems

Karlsruhe, Germany

lars-fredrik.berg@ict.fraunhofer.de

\begin{abstract}
The implementation of electric drivetrains into passenger vehicles is one of the promising ways for the automotive industry to reduce $\mathrm{CO}_{2}$ fleet emissions. The most important aim for the current developments is to increase range and performance while assuring affordability for the customer. In the field of electric motor development for traction applications, great efforts are necessary in order to improve electrical machines in terms of efficiency, power density and costs.
\end{abstract}

The optimization of each individual field is a subject of research. Typically, there is a conflict of interest in simultaneously optimizing efficiency, power density and costs. This work presents a new approach to optimize the three fields for electric traction motors. The new approach combines an efficient direct cooling concept with the possibility of using lightweight polymer composites for the electric motor housing. The cooling concept increases the efficiency in a wide range of operation while enabling a high maximum continuous power output from the motor. To estimate the potential of the used cooling topology, the winding is optimized for using stator slot cooling. The electric motor is thermally simulated to verify the concept. These findings are used to design the cooling channels. Finally, a molded prototype stator is built and the newly designed concept was validated in a component test setup.

The direct cooling with its short thermal path between the area of heat generation to the cooling system, enables the use of thermally insulating thermosetting composite materials for the electric motor housing. In this work the feasibility and potential of manufacturing the stator housing of an electric motor in an injection molding process is investigated. The design freedom of this manufacturing process enables complex and extensive functional integration such as the direct incorporation of the cooling channels in the stator slots, the phase connectors and the coolant supply. Key
density
Keywords-thermoset molding, stator slot cooling, high power

\section{INTRODUCTION}

Many applications such as electrical cars or aviation applications raise a demand for electrical machines with high power density, high efficiency and low material and production costs. As a result of higher power density, electrical machines get lighter. Less weight and higher efficiency lead to an extended vehicle range compared to standard machines. Furthermore, by saving weight, the material costs can often be reduced. Additional costs can be reduced, if manufacturing processes suitable for a high volume production are used. The potential of this approach is to be examined here.

The starting point of this work is an electrical machine optimized with regard to power density, see the properties in Table 1. The properties are calculated in a finite-elementmethod (FEM) simulation. As shown in [1] efficiency and power density are competitive objectives, though. Copper losses in stranded wires are proportional to

$$
P_{\mathrm{L}, \mathrm{Cu}} \sim \frac{\rho_{\mathrm{Cu}, \mathrm{amb}}\left(1+\alpha_{\mathrm{Cu}}\left(\vartheta_{\mathrm{Cu}, \mathrm{avg}}-\vartheta_{\mathrm{amb}}\right)\right)\left(L_{\mathrm{Fe}}+L_{\mathrm{EW}}\right)}{A_{\mathrm{Slot}} \cdot k_{\mathrm{f}}}
$$

where $P_{\mathrm{L}, \mathrm{Cu}}$ are the copper losses, $\rho_{\mathrm{Cu}, \mathrm{amb}}$ the electric specific resistance at ambient temperature $\vartheta_{\mathrm{amb}}, \alpha_{\mathrm{Cu}}$ the temperature coefficient of electrical resistance, $A_{\text {Slot }}$ is the cross-sectional copper area, $L_{\mathrm{Fe}}$ and $L_{\mathrm{EW}}$ are the stator lamination and end-winding length, $k_{\mathrm{f}}$ the electrical copper filling factor and $\vartheta_{\mathrm{Cu} \text {,avg }}$ the average copper temperature. 
Table 1: Properties of the synchronous machine

\begin{tabular}{ll}
\hline \multicolumn{1}{c}{ Property } & \multicolumn{1}{c}{ Value } \\
\hline Wire & Flat Wire \\
Electrical Filling Factor & $54.5 \%$ \\
Poles / Slots & $8 / 12$ \\
Active axial Length & $80 \mathrm{~mm}$ \\
Rated Current & $200 \mathrm{~A}$ \\
Rated Power & $50 \mathrm{~kW}$ \\
Rated Torque & $48 \mathrm{Nm}$ \\
Rated Speed & $10000 \mathrm{rpm}$ \\
Overall losses at rated OP & $3.418 \mathrm{~kW}$ \\
Stator Iron losses at rated OP & $0.372 \mathrm{~kW}$ \\
Copper losses at rated OP & $2.503 \mathrm{~kW}$ \\
Efficiency at rated OP & $93.6 \%$ \\
Estimated Total Weight & $12,3 \mathrm{~kg}$ \\
\hline
\end{tabular}

A reduction of the copper losses without increasing the copper surface can be achieved by increasing the fill factor. To increase the fill factor, more complex winding techniques must be used, which can lead to higher production costs. Advanced cooling concepts are needed to lower the average copper temperature.

One possible way to increase the copper fill factor is to use rectangular flat wires in single-tooth winding machines. This winding process can be automated with a modified linear winding machine [2]. Using rectangular flat wires and stator teeth with parallel side surfaces lead to unusable triangular spaces between two winding phases, which can be used for cooling channels. As shown in Fig. 2, the cooling channels replace the traditional water cooling jacket. With the use of rectangular flat wire additional eddy current losses within the windings come along. Therefore, an optimized winding arrangement was used below [1].

Using the space between two winding phases as cooling channels shortens the thermal path and increases the cooling capacity [3]. The effect of an enhanced cooling and fill factor on the copper losses is shown in Fig. 1. Both measures result in reduced copper losses hence increased efficiency.

Conventionally, the alloy housing ensures both stability and the cooling path from the winding to the cooling jacket. Fig. 2 compares the conventional with the new approach. This new approach decouples the need for stability from the need for cooling performance. It allows the use of lightweight polymer composites for the housing and the design of advanced cooling technologies with manufacturing processes, that are suitable for mass production. This increases efficiency, power density and reduces costs.

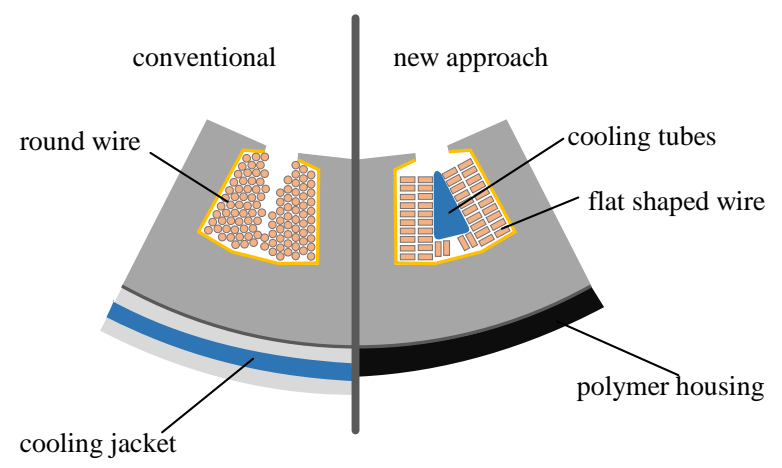

Fig. 2: Comparison of the conventional cooling jacket (left) to the stator slot cooling (right)

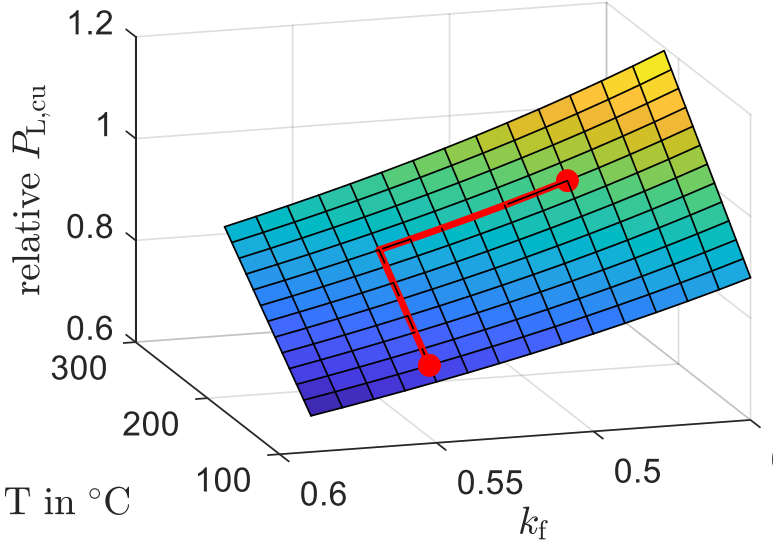

0.45

Fig. 1: Relative effect of $k_{f}$ and $\vartheta_{C u, \text { avg }}$ on $P_{L, C u}$ originating from

$$
k_{f, 1}=0.49 \text { and } \vartheta_{C u, a v g, 1}=180^{\circ} \mathrm{C}
$$

Due to the decoupling, the housing is made out of a different material, than the cooling channels. The advanced design of the cooling channels and further benefits of the used manufacturing processes are presented in II.A. The characteristics of the used materials are described in II.B. The underlying thermal and structural simulations are explained in II.C. Influence on the cooling performance of manufacturing tolerances are discussed in II.D. The presented approach is validated in III.

\section{Design AND Simulation}

\section{A. Introduction of the Design}

The motor, designed for traction applications, needs a minimum of connections to allow fast assembly into a vehicle. To adjust to the necessity for a tight packaging, only one coolant connector for supply is desirable. To achieve a preferably dense packing, the coolant exits on the same side of the engine as it enters. This is realized by two cooling channels in series and six of these in parallel, as a total of twelve cooling channels. The coolant is provided via an inner ring-tube and collected via another outer ring-tube, as shown in Fig. 3 .

To balance the flow through the cooling channels, the connection of the cooling intakes is slightly different in their cross-sectional area. To determine the necessary crosssectional area of each intake, a computational fluid dynamics (CFD) analysis is made. To guarantee the cooling of the winding heads, the ring-tubes as well as the intakes are close enough to provide cooling. For the A-bearing shield, the cooling channel overtops the intake. This creates a turbulent flow in the cooling, but also provides a close connection of the winding heads to the coolant. As injection molding is a

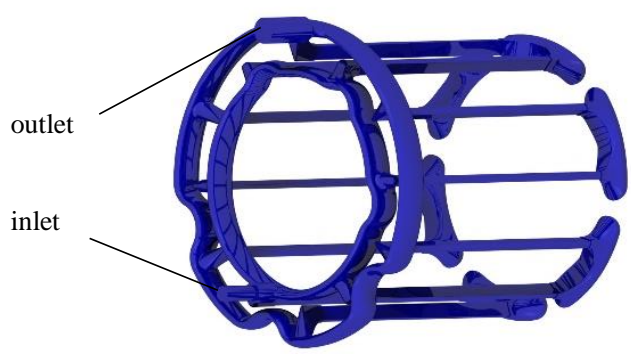

Fig. 3: Ring-tube with twelve cooling channels, six pairs in parallel 
process that can only produce geometrical undercuts with additional effort, the cooling channels are created by the assembly of housing and stator. Two systems exist for the sealing of the two parts. For sealing to the rotor gap and the outer part of the stator, in each case one O-ring is used. To seal the high-pressure and the low-pressure ring-tube, only a labyrinth seal is used. Small leakage is acceptable for small design space. For the torque transmission between the stator and the housing there are twelve slots in the stator with corresponding keys in the housing.

Fig. 4 pictures the phase connector. As the housing material is electrically nonconductive, it will be utilized for housing of the phase connectors. Because of the way of assembling the stator into the housing, the phase connector must not have any undercut. To achieve the necessary copper cross-sectional, which should be at least of the same size as the windings, a round connector would have required more space than available, or would reach a too high length. Further, a simple shape for best sealing during molding was desirable. The connector on the stator side is a simple rectangular shape, so it is possible to utilize the copper rings that distribute the current to the windings. This avoids additional work like crimping, which would increase the resistance. The connector housing is integrated in the motor housing. Through a union nut and a wave spring the pin is pressed onto a ramp and into the phase connector. Through the ramp a mechanical advantage of one to five is realized to reduce the resistance. When plugging the connector, the pin moves downwards during contact. This helps with surface oxidation, which is broken up.

To further reduce the number of connections, a printed circuit board (PCB) will be integrated into a lid. Fig. 5 pictures the lid which forms an additional cooling channel for a possible rotor cooling, as well as it connects to the statortemperature sensors and the rotor infrared sensor and feeds through the signal of the rotor position encoder. The temperature readings will be transferred out via a controller area network bus system. With the feedthrough of the position encoder, a single data connector is sufficient for the whole motor. To seal and improve the robustness of the PCB, it is overmolded into the lid. The lid itself is bonded to the housing. Additionally, it is secured with three self-tapping screws, to allow a fast assembly of the motor.

Table 2: Material data of the used polymers

\begin{tabular}{lll}
\hline \multicolumn{1}{c}{ Material } & \multicolumn{1}{c}{ Use } & Thermal conductivity \\
\hline EME-A730E & potting & $3 \mathrm{~W} /(\mathrm{m} \cdot \mathrm{K})$ \\
steel & bundle of lamination & $15 \mathrm{~W} /(\mathrm{m} \cdot \mathrm{K})$ \\
copper windings & coils (half of $\mathrm{Cu})$ & $175 \mathrm{~W} /(\mathrm{m} \cdot \mathrm{K})$ \\
\hline
\end{tabular}

\section{B. Used manufacturing Processes}

As described above, the stator consists of two polymer parts, between which the cooling channels are formed. The materials used for these parts are tailored to the specific requirements of the housing and the stator potting (Table 2).

The housing has to provide mechanical strength and stiffness to the assembly. Furthermore, it protects all electrical active parts from external influences. Since the heat is taken away directly at the copper windings, the housing material itself does not need to be heat conductive. Furthermore, it needs to be resistant to automotive fluids, especially water-glycol coolants.

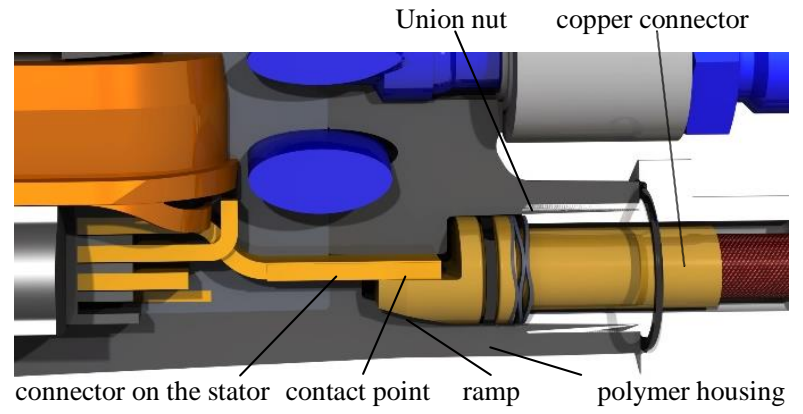

Fig. 4: CAD model of the phase connector, enclosed in the housing

The material of choice for these requirements is a thermosetting phenolic molding compound, which exhibits excellent mechanical properties at elevated temperatures because of the chemical crosslinking. For the housing, a glass fiber reinforced phenolic molding compound by Sumitomo Bakelite, Co. (Vyncolit X7700) is used. The material is processed via injection molding, ensuring high reproducibility, dimensional accuracy and short cycle times.

The main requirement for the stator potting material is a low viscosity during the mold filling phase, hence all electronic active parts are overmolded without being damaged. Since a small layer of the potting material ensures the electrical insulation between the cooling channels and the copper winding, a certain heat conductivity is required. The mechanical requirements for the potting material are lower, because the part is completely encapsulated and supported by the outer housing.

The low viscosity during the mold filling phase can typically only be achieved by thermosetting materials. Due to a highly filled epoxy molding compound by Sumitomo Bakelite, Co. (EME-A730E) was used. The main filler is aluminum oxide, which results in a thermal conductivity of $3 \mathrm{~W} /(\mathrm{m} \cdot \mathrm{K})$. Because of the low viscosity, a transfer molding process is used for molding the stator potting. All electrical components are preheated and positioned in the mold. Due to the low resin viscosity during the mold filling phase, the electrical components are gently overmolded without being damaged.

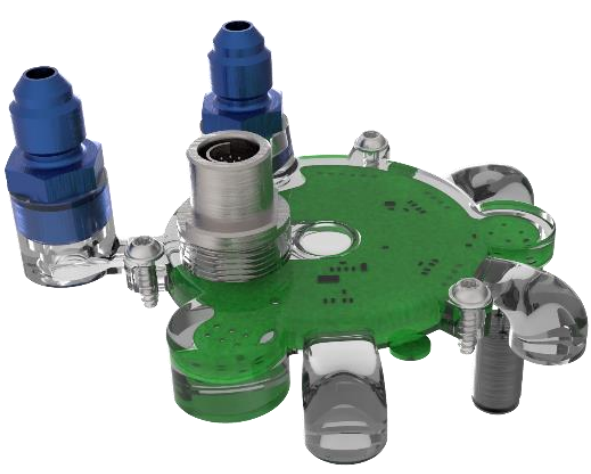

Fig. 5: Electric lid: overmolded PCB with additional cooling channel for possible rotor cooling 


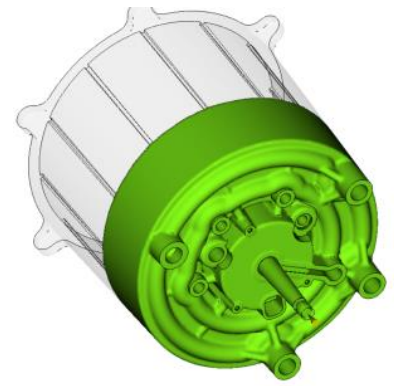

(a)

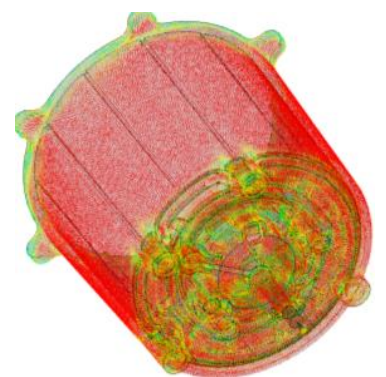

(b)

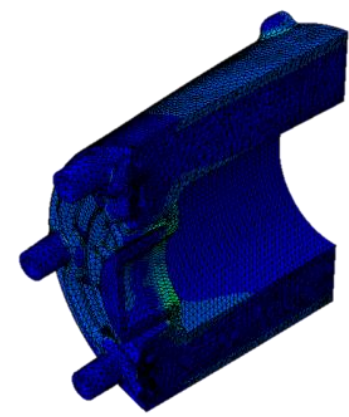

(c)

Fig. 6: Simulation of the housing along the CAE-Chain. Mold filling Simulation (a), Fiber orientation for mapping (b) and anisotropic structural FEM-analysis (c).

\section{Simulation of Design}

To validate the design of the housing, a combination of process and structural simulation is done along the CAEchain [4]. Mold filling simulations with Moldflow 2018.1 (Autodesk, San Rafael, CA, USA) and OpenFOAM 4.1 (OpenCFD Ltd., Bracknell, UK) are carried out to optimize the design with respect to air traps, weld lines, filling behavior and fiber orientation. The used material models and the used OpenFOAM code are the same as descripted in [5].

To perform a realistic structural analysis, a mold filling simulation is done (Fig. 6a). The process-induced fiber orientation (Fig. 6b) calculated in this filling simulation is exported and mapped to an FEM mesh (Fig. 6c). With the mapped data an anisotropic structural analysis is done with ABAQUS to verify required the stiffness und strength of the housing. The needed housing stiffness for bearings are achieved.

The cooling concept is further analyzed by simulating the thermal characteristics to discover hotspots, get an overview of the general thermal behavior and to give insights, which process developments should be considered for further investigations. Therefore, the stator is modeled including the

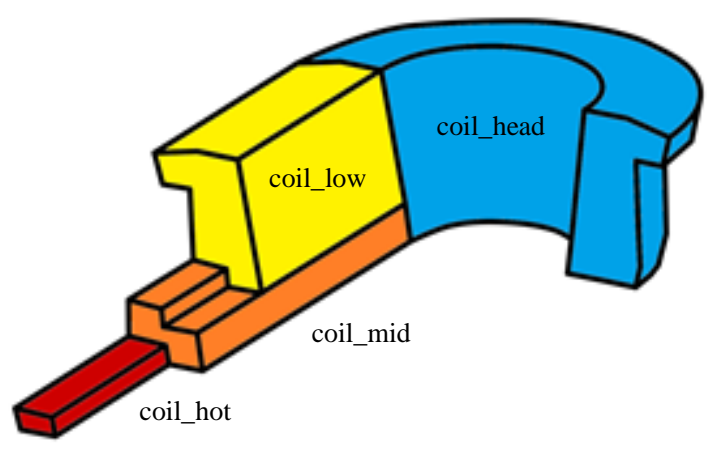

Fig. 7: Division of the coil by applied energy density bundle of laminations, the coils and the surrounding potting with the material parameters from Table 2.

The values of Table 3 are applied volumetrically to the metal parts. The coils are divided to take into account their very inhomogeneous energy distribution as shown in Fig. 7. The energy distribution results from a magnetic FEM simulation in the operating point described in Table 1. The boundary conditions are modeled as a water/stator potting heat transfer with a evenly distributed cooling water temperature of $80^{\circ} \mathrm{C}$. The used thermal conductivities are listed in Table 2. All other boundaries are presumed to be adiabatic. The simulation shows the thermal equilibrium that is established after a long time. As discussed above most of the assumptions made for the model are conservatively.

Fig. 8a and Fig. 8b show that the intended cooling near the coils is established effectively. The hotspots in Fig. 8c are on the outer surface of the bundle of laminations and underline this conclusion. In Fig. 8 the water/stator potting heat transfer is set to $5 \mathrm{~kW} /\left(\mathrm{m}^{2} \cdot \mathrm{K}\right)$. This results in a maximum temperature of $180^{\circ} \mathrm{C}$. The simulation is designed so that all temperatures can be considered as differential temperatures. That means the hotspot is $100 \mathrm{~K}$ hotter than the cooling water.

Table 3: Power Distribution

\begin{tabular}{lrrr}
\hline & $\begin{array}{c}\text { Energy Density } \\
\text { in W/mm }\end{array}$ & $\begin{array}{c}\text { Energy in } \\
\mathrm{W}\end{array}$ & $\begin{array}{c}\text { Volume in } \\
\mathrm{mm}^{3}\end{array}$ \\
\hline bundle of lamination & 0.68 & 372.00 & 545393.69 \\
coil_head & 13.64 & 600.00 & 43997.64 \\
coil_hot & 37.29 & 261.60 & 7016.04 \\
coil_mid & 18.90 & 499.20 & 26419.56 \\
coil_low & 13.21 & 1142.28 & 86445.12 \\
\hline sum & - & 2875.08 & 709272.05 \\
\hline
\end{tabular}
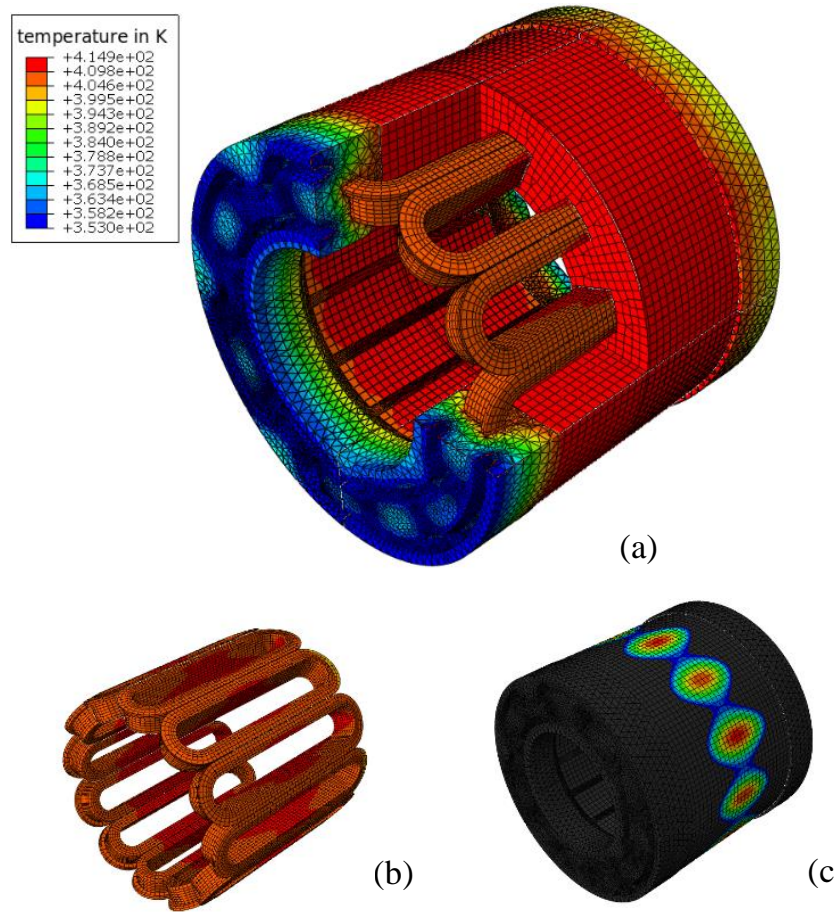

Fig. 8: Simulation temperature results in $K$ at $0.6 \mathrm{~mm}$ wall thickness and $5 \mathrm{~kW} /\left(\mathrm{m}^{2} \cdot K\right)($ a) cutaway showing the general temperature distribution (b) temperature distribution and hotspots on the coils $(c)$ global hotspots identified in the simulation (different legend) 


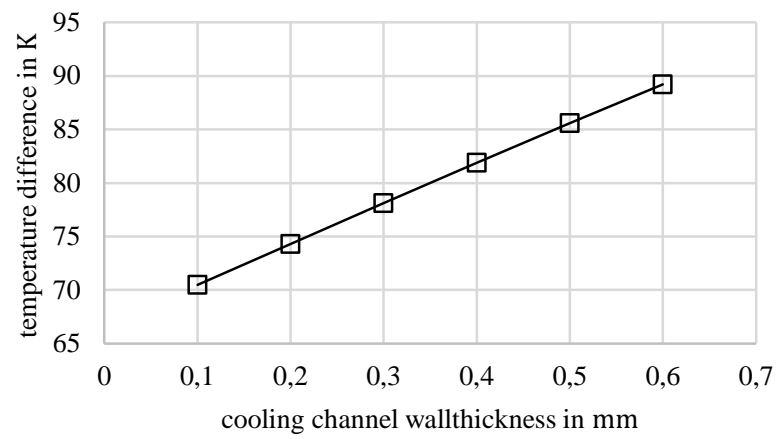

Fig. 9: Difference in cooling water inlet temperature and maximum of bundle of lamination temperature over varied cooling channel wall thickness

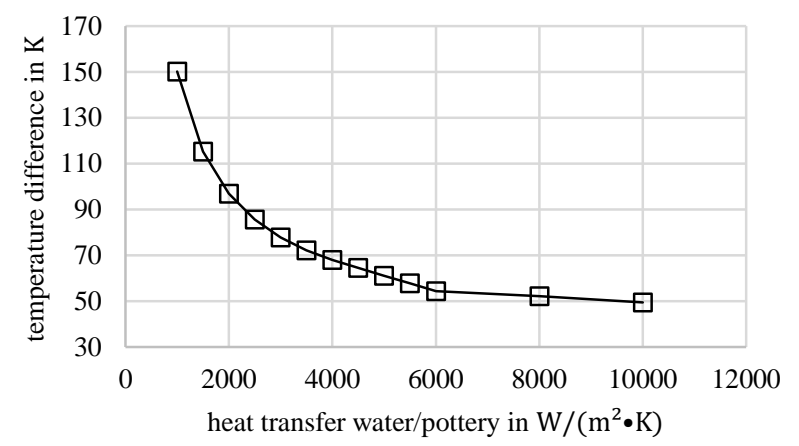

Fig. 10: Difference in cooling water inlet temperature and maximum of bundle of lamination temperature over varied water/potting heat transfer

\section{Sensitivity study on cooling performance}

To gain further insights on the influence of production tolerances and to gain options for further process optimization, the sensitivity of maximum temperature to wall thickness is investigated. Therefore, the wall thickness is varied between $0.1 \mathrm{~mm}$ and $0.6 \mathrm{~mm}$ in Fig. 9 .

Also the influence of heat transfers between water and the potting is varied. Fig. 10 shows an exponential influence on the maximum temperature of the electric engine. In comparison to the wall thickness, the heat transfer between water and potting has a much higher range and therefore a bigger sensitivity. The biggest gain of efficiency can be made by tuning the water/stator potting boundary.

\section{CONCEPT VALIDATION}

This chapter shows the cooling performance of the overmolded stator potting. The components test setup consists of a temperature control unit, several temperature, power and pressure measuring instruments as well as a power inverter. A three-phase inverter is used to produce symmetrical losses in the star-connected stator.

\section{A. Component Test Setup}

In the stator potting, the inverter generates an alternating current and field that generates copper and iron losses. Because of the missing rotor, the predominant losses are copper losses. The generated losses are measured with a Yokogawa wt3000 precision power analyzer directly on the stator. Since no electrical energy is converted into mechanical energy, the measured power $P_{\mathrm{L}}$ is a pure power loss consisting of copper and iron losses.
Due to the missing rotor, the actual magnetic conditions cannot be reproduced. Therefore, the total losses to be cooled consist of copper and iron losses: $P_{\mathrm{L}, \text { tot }}=2.503 \mathrm{~kW}+$ $0.372 \mathrm{~kW}=2.875 \mathrm{~kW}$ with respect to Table 1 . It is assumed that the rotor is thermally decoupled, since on the one hand it has a rotor cooling and, on the other hand, the air gap, including a plastic layer, has an insulating effect. Hereinafter, no distinction is made between copper and iron losses, but only the total losses are indicated.

The temperature control unite provides a constant water flow at a constant temperature. To inject as much losses $P_{\mathrm{L}}$ as possible and remain below critical stator potting temperature, a water inlet temperature of $22.9^{\circ} \mathrm{C}$ is used for this test setup. Since the simulation considers differential temperatures, this has no influence on the comparability between simulation and measurement.

\section{B. Measurement of cooling performance}

The cooling channels of the stator potting are designed for a pressure of up to $2 \mathrm{bar}$. The measurements were taken at a flow rate of $\dot{V}=12 \mathrm{l} / \mathrm{min}$. The system pressure of $1.95 \mathrm{bar}$ was measured between the outlet of the temperature control unit and the inlet of the cooling channels, Fig. 3. No visible leakage occurred. Three temperature sensors were evenly distributed over the circumference of the bundle of stator lamination.

The maximum temperature of the three sensors is shown in Fig. 11 as a function of the electrical power $P_{\mathrm{L}}$. With increasing power $P_{\mathrm{L}}$, the temperature rises. At a heat input of $P_{\mathrm{L}, \max }=2.352 \mathrm{~kW}$, the stator temperature remains below $148{ }^{\circ} \mathrm{C}$. The difference of the cooling water inlet $\left(22.9^{\circ} \mathrm{C}\right)$ and outlet $25.51{ }^{\circ} \mathrm{C}$ temperature showed that about $93 \%$ of the electrical power $P_{\mathrm{L}}$ was removed by the cooling water. The remaining $7 \%$ are removed due to heat radiation and natural convection.

\section{Discussion of Measurement}

Since the maximum temperature of the housing was reached before the desired power dissipation $P_{\mathrm{L}, \text { tot }}$ could be generated, the measurement cannot be applied directly to the simulation. Nevertheless, the measurement is compared with the simulation using a linear fit, see Fig. 11. At the desired total power loss of $P_{\mathrm{L}, \text { tot }}=2.875 \mathrm{~kW}$ the stator would have a temperature of $164.5^{\circ} \mathrm{C}$. This means that the bundle of stator lamination is $141.6 \mathrm{~K}$ warmer than the cooling water. In comparison with the simulation of Fig. 10, the stator would have a heat transfer rate in the cooling channel of $1122 \mathrm{~W} /\left(\mathrm{m}^{2} \cdot \mathrm{K}\right)$.

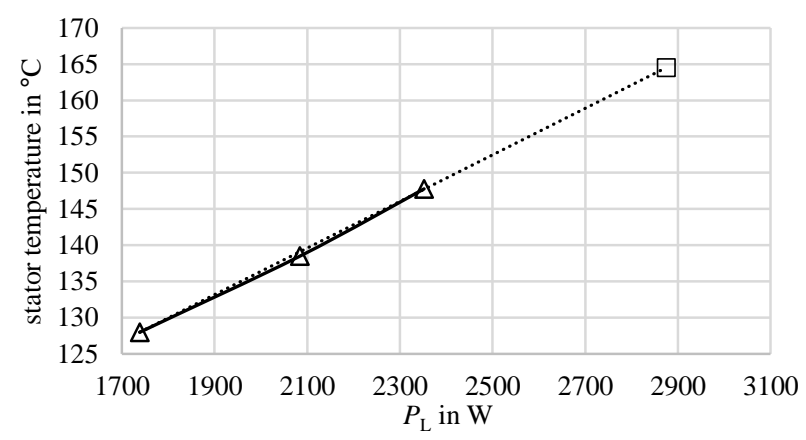

Fig. 11: Stator temperature over $P_{L}$, triangular measured temperature, linear extrapolate temperature 
Starting from this point, the temperature can be lowered by the two mechanisms described in II.D. The temperature can be lowered either by thinner cooling channel walls or by increasing the heat transfer coefficient.

Currently, the cooling channel wall is designed to be $0.6 \mathrm{~mm}$ thick due to restrictions in the production technology. By improving the production tolerances of the winding, the thickness of the cooling channel wall is expected to be reduced to $0.1 \mathrm{~mm}$. As simulated and illustrated in Fig. 9, this would decrease the temperature of the bundle of lamination by approximately $20 \mathrm{~K}$.

Increasing the heat transfer coefficient, as shown in Fig. 10 , could also decrease the temperature of the bundle of lamination by approximately $90 \mathrm{~K}$. A better heat transfer between water and cooling channel could be achieved by improving the sealing and increasing the volume flow. In addition, further mechanical changes in the cooling channel might lead to a more turbulent distribution of water.

Overall, there is still a potential reduction of $110 \mathrm{~K}$ in the above mentioned improvements. In this publication it was found, that it is possible to build a stator potting with an integral body and stator slot cooling system using manufacturing processes suitable for high volume production. With this first prototype, the desired cooling capacity to drive the rated operation point from Table 1 is achieved by $82 \%$. With the data of the synchronous machine from Table 1 this would result in a power density of $3.28 \mathrm{~kW} / \mathrm{kg}$.

\section{CONCLUSION}

In this work a new concept for the high volume production of high power density synchronous machines with concentrated single-tooth winding was presented. It was discussed which mechanisms enable a thermally isolated machine to work. An internal stator cooling is used to avoid the need for a thermal path through the housing. Thus, the properties of the conventional housing could be decoupled in a mechanically stabilizing and in a heat conducting part. This was enabled by the use of thermoset materials.

At the same time, it was shown that new possibilities in the design of the cooling concept are accompanied by the use of new materials and the associated processing. Complex cooling channels in the stator slot could be molded directly during the manufacturing process. The presented concept promises possible higher power density, higher efficiency for many operating points and a lower price, due to the lower manufacturing costs of the illustrated processing.
Furthermore, the parameters, which have a major impact on the cooling performance, have been identified. In a further development, these can be specifically addressed. Tighter winding tolerances, increased coolant mixing and higher flow rates can further increase the efficiency of the electrical machine.

Future work will cover the overall concept. First, the performance of the electric machine including the rotor is validated. Thereafter, further investigations with regard to continuous load resistance, NVH, bearing currents can be carried out.

The development can have a significant impact on the automotive industry, which relies on high efficiency and therefore high range. Both longer range and cost-effective electric motors are important factors to increase the market penetration of electric vehicles.

\section{ACKNOWLEDGMENT}

We would like to thank the Vector Foundation, Stuttgart, Germany for the financial support of the project. Also we would like to thank SBHPP for providing material and technique. In addition, we would like to thank those responsible at KIT and Fraunhofer ICT, who facilitate such interdisciplinary and joint research.

\section{REFERENCES}

[1] M. Schiefer and M. Doppelbauer, "Indirect slot cooling for highpower-density machines with concentrated winding," Proc. - 2015 IEEE Int. Electr. Mach. Drives Conf. IEMDC 2015, pp. 1820-1825, 2016.

[2] J. Hagedorn, "Vorrichtung und Verfahren zum Wickeln eines Flachdrahtes," 2009

[3] M. Gröninger, F. Horch, A. Kock, M. Jakob, and B. Ponick, "Cast coils for electrical machines and their application in automotive and industrial drive systems," in 2014 4th International Electric Drives Production Conference, EDPC 2014 - Proceedings, 2014.

[4] L. Kärger et al., "Development and validation of a CAE chain for unidirectional fibre reinforced composite components," Compos. Struct., vol. 132, pp. 350-358, Nov. 2015.

[5] F. Wittemann, R. Maertens, A. Bernath, M. Hohberg, L. Kärger, and F. Henning, "Simulation of Reinforced Reactive Injection Molding with the Finite Volume Method," J. Compos. Sci., vol. 2, no. 1, p. 5, Jan. 2018. 